\title{
Editorial
}

\section{Pediatric Nephrology: An Update}

\author{
Amin J. Barakat ${ }^{*} \S$
}

Georgetown University Medical Center, Washington, DC, USA

I am indeed privileged to serve as Guest Editor to this special issue of the Open Urology and Nephrology Journal on pediatric nephrology. I am also appreciative to the distinguished panel of authors for their contribution and for presenting the most recent clinical advances in various aspects of the specialty.

Pediatric nephrology is rapidly evolving. Over the last few decades we have witnessed a rapid change in nosology, etiology, pathogenesis and treatment of renal disease. We have seen advances in renal and urinary tract imaging as well as more precise use of novel urinary biomarkers to define the type and degree of renal injury, both acute and chronic. We now have better understanding of factors contributing to normal and abnormal renal development, particularly the progenitors of renal development and regeneration, delivery of progenitor cell therapeutics, and the role of epigenetics [1]. Multi center pan-national and international clinical studies have helped to put together clinical guidelines to diagnose and manage renal disease. In this issue, we will discuss few, but important recent clinical developments in pediatric nephrology.

While performing renal ultrasound and voiding cystourethrogram (VCUG) was once a routine, the American Academy of Pediatrics (AAP) and the National Institute for Health and Clinical Excellence (NICE) are now recommending a marked reduction in the imaging of children under age 2-3 with febrile urinary tract infection (UTI) [2]. Both AAP and NICE also agree that prophylactic antibiotic treatment should not be routinely used in these children even those with major vesicoureteral reflux (VUR).

Presently, the AAP recommends that infants (2-24 months of age) with a febrile UTI undergo renal and bladder ultrasonography as a routine screen, and reserve VCUG for patients with recurrent UTI or with evidence of anatomic abnormality on ultrasound. In 2014, and only three years after this recommendation, the results of the Randomized Intervention for Children with Vesicoureteral Reflux (RIVUR) study was published [3]. According to this study, antibiotic prophylaxis does indeed prevent UTI recurrence in young children with VUR, but at the cost of increased antibiotic resistance, and with unclear benefits in preventing

*Address correspondence to this author at the 107 North Virginia Avenue, Falls Church, VA 22046, USA; Tel: 703-532-4446; Fax: 703-532-8426;

E-mail: aybarakat@aol.com

${ }^{\S}$ Guest Editor underlying renal damage. Genetic factors may be involved in the risk for UTI, and genetic markers may become available in the future to help in more precise selection of children at risk for scarring, thus helping in identifying patients who need treatment with antibiotics.

Despite extensive research, the debate regarding how to screen and treat patients with VUR continues. Most clinicians use renal and bladder ultrasound followed by a VCUG, when indicated. Concerns regarding the invasiveness of VCUG and spontaneous resolution of VUR have led to an alternative "top-down" approach which employs a DMSA scan soon following a febrile UTI. A VCUG would be performed on patients with focal photopenia on DMSA. The rationale for this approach is that only VUR resulting in pyelonephritis is clinically relevant [4]. Recent studies also stress the role of bladder and bowel dysfunction (BBD) in the development of recurrent febrile UTI and potential subsequent renal scarring. Successful BBD management promotes downgrading or elimination of VUR.

The debate of antibiotic prophylaxis in recurrent UTI and VUR in children remains unresolved, and the papers of Drs. Finnell and Rensing address this issue in detail. Further research is needed to determine the most effective way to evaluate pediatric patients after their first febrile UTI.

The economic and social burden of diagnosing, treating and preventing chronic kidney disease (CKD) in children remains substantial. There have been many advances in identifying factors that predict development of CKD and its progression, as well as in the management of co-morbid conditions. Recent reports from retrospective registry data analysis and multi-center prospective studies have significantly advanced our knowledge in the management of $\mathrm{CKD}$ and the pharmacological treatment of renal transplantation. Despite advances in the understanding of the pathogenesis, diagnosis and treatment of CKD, much work remains to be done to improve the long term outcome of this disease. Given the complex medical issues associated with $\mathrm{CKD}$, it is important that pediatric nephrologists and urologists work together with the pediatrician to optimize the medical care of these patients.

While hypertension (HT) in children was previously assumed to be secondary to renal, cardiovascular and endocrine causes, we now know that it is mostly a part of the spectrum of essential HT mainly linked to the obesity epidemic. The relationship of HT to overweight and obesity has been documented worldwide. Essential HT now accounts for over $85 \%$ of HT between the ages of 12 and 18. Several 
studies including a recent meta regression analysis indicate clearly that blood pressure in childhood tracks into adulthood [5]. Pediatricians and health care professionals dealing with children can, and should play a pivotal role in the early diagnosis and treatment of HT to reduce long-term cardiovascular morbidity and mortality.

Prenatal diagnosis of congenital anomalies of the kidney and urinary tract (CAKUT) has also seen advances. In 2014, a consortium of healthcare providers including maternal fetal medicine physicians and pediatric radiologists, nephrologists, and urologists had consensus on the classification of prenatal and postnatal urinary tract dilation (UTD) [6]. The use of terms such as hydronephrosis and caliectasis, were discouraged. While ultrasound is a reasonable screening tool for the antenatal detection of UTD, it cannot reliably determine its underlying etiology at the present time. Although sonographic assessment of renal appearance and amniotic fluid volume, as well as urinary electrolyte analysis, are the most useful predictors of preserved renal function in fetal lower urinary tract obstruction (LUTO), they too are limited in their ability to predict outcomes on an individual basis. Data evaluating the efficacy of vesicoamniotic shunt therapy for the treatment of fetal LUTO continue to be inconclusive. Dr. Miller's paper discusses fetal LUTO in a comprehensive way and presents limitations of prenatal diagnosis and fetal therapy.

The most impressive advances in pediatric nephrology; however, have been in the genetics of renal disease. Since Reeders et al. [7] described the linkage marker for ADPKD to be closely linked to the alpha-globin locus on the short arm of chromosome 16 in 1985, the knowledge of genetics of kidney disease has exploded. The number of genes associated with renal disease is increasing exponentially, leading to a clearer understanding of its pathophysiology. It is also appreciated now that a genetic mutation(s) underlie many renal syndromes. Mutations in several renal development genes have been identified as causes for congenital anomalies of CAKUT. In spite of technical and ethical issues at the present time, genetic testing offers the possibility to diagnose some renal diseases without the need for a renal biopsy and allows the prenatal diagnosis of certain renal diseases in at-risk fetuses, or identification of potential renal disease before it has become manifest. Identification of a specific gene mutation holds the possibility of correction though gene therapy, although this is mostly experimental at the present time. Genes have now been identified in nephrotic syndrome, hereditary nephritis, lipoprotein glomerulopathy, glomerular mitochondrial disorders, renal tubular disease, hypertension, cystic kidney diseases, nephronophthesis and many syndromes associated with CAKUT. As Dr. Foreman states in his excellent paper in this issue, " In the future, genetic testing will become as easy and as common as ordering a serum creatinine is today."

Pediatricians and healthcare providers who treat children should stay current on the pediatric, nephrology and urology literature as the science is continuously evolving.

\section{REFERENCES}

[1] Chesney RW, Checalier R. Thirty-three years of progress: the international workshops on developmental nephrology and the role of IPNA. Pediatr Nephrol 2014; 29: 499-504.

[2] Tullus K. What do the latest guidelines tell us about UTIs in children under 2 years of age. Pediatr Nephrol 2012; 27: 509-11.

[3] Hoberman A, Greenfield SP, Mattoo TK, et al. Antimicrobial prophylaxis for children with vesicoureteral reflux. N Engl J Med 2014; 370: 2367-76.

[4] Hansson S, Dhamey M, Sigström, O, et al. Dimercapto-succinic acid scintigraphy instead of voiding cystourethrography for infants with urinary tract infection. J Urol 2004; 172: 1071-3.

[5] Cheng X, Wang Y. Tracking blood pressure from childhood to adulthood: a systematic meta-regression analysis. Circulation 2008; 117: 3171-80.

[6] Nguyen HT, Benson CB, Bromley B, et al. Multidisciplinary consensus on the classification of prenatal and postnatal urinary tract dilation (UTD classification system). J Pediatr Urol 2014; 10: 982-98.

[7] Reeders ST, Breuning MH, Davies KE, et al. A highly polymorphic DNA marker linked to adult polycystic kidney disease on chromosome 16 . Nature $1985 ; 317$ : 542-4. 